Bull. Korean Math. Soc. 51 (2014), No. 4, pp. 1031-1040

http://dx.doi.org/10.4134/BKMS.2014.51.4.1031

\title{
A NOTE ON CUBICALLY HYPONORMAL WEIGHTED SHIFTS
}

\author{
Chunji Li, Muneo Chō, And Mi Ryeong Lee
}

\begin{abstract}
In this paper, we show that any cubically hyponormal weighted shift with first two equal weights is flat. And we give an example of a weighted shift which is not cubically hyponormal but almost-cubically hyponormal.
\end{abstract}

\section{Introduction and preliminaries}

Let $\mathcal{H}$ be a separable, infinite dimensional, complex Hilbert space and let $\mathcal{L}(\mathcal{H})$ denote the algebra of all bounded linear operators on $\mathcal{H}$. An operator $T$ is polynomially hyponormal if $p(T)$ is hyponormal for all (complex) polynomials $p$ (cf. [6]). And an operator $T$ in $\mathcal{L}(\mathcal{H})$ is weakly n-hyponormal if $p(T)$ is hyponormal for any polynomial $p$ with degree $n$ or less (cf. [8]). In particular, the weak 2-hyponormality (or weak 3-hyponormality) referred to as quadratic hyponormality (or cubic hyponormality, resp.) has been considered in detail in [4], [5] and [8]. It is well known that "subnormal $\Rightarrow$ polynomially hyponormal $\Rightarrow \cdots \Rightarrow$ weakly 3 -hyponormal $\Rightarrow$ weakly 2 -hyponormal $\Rightarrow$ hyponormal". However, one does not know about converse implications for $n \geq 3$ yet; see [2], [5], [8] for weak 2- and weak 3-hyponormalities.

A unilateral weighted shift is often used to study the bridges between subnormality and hyponormality. In [9] Stampfli proved that every subnormal weighted shift $W_{\alpha}$ with any two equal weights has flatness, i.e., if $\alpha_{k}=\alpha_{k+1}$ for some $k \in \mathbb{N}_{0}=\mathbb{N} \cup\{0\}$, then $\alpha_{1}=\alpha_{2}=\cdots$. In [2], R. Curto proved that the 2-hyponormal weighted shift $W_{\alpha}$ with any two equal weights has flatness. Also he obtained a weighted shift $W_{\alpha}$ with first two equal weights without being flat. Moreover, he raised a question: describe all quadratically hyponormal weighted shifts with first two equal weights, which was studied as several kinds of detections. In [1], Y. Choi proved that every polynomially hyponormal weighted

Received August 11, 2013.

2010 Mathematics Subject Classification. Primary 47B37; Secondary 47B20.

Key words and phrases. weighted shift, cubically hyponormal operator, flatness.

The first author was supported by the National Science Foundation of China under Grant 11171301, and the second author was supported by Grant-in-Aid Scientific Research No. 24540195 . 
shift $W_{\alpha}$ with any two equal weights has flatness. In particular, he showed that if $W_{\alpha}$ is a weighted shift with first two equal weights and $W_{\alpha}+s W_{\alpha}^{n}$ is hyponormal for all $s \in \mathbb{C}$ and all $n \in \mathbb{N}$, then $W_{\alpha}$ is flat. In this paper, we show that any cubically hyponormal weighted shift $W_{\alpha}$ with first two equal weights is flat (see Theorem 3.3 below). Also, we give an example of a weighted shift which is not cubically hyponormal but almost-cubically hyponormal (see Definition 2.1 below).

Some of the calculations in Section 3 were obtained through computer experiments using the software tool Mathematica [10].

\section{Constructions of formulas}

For $A, B \in \mathcal{L}(\mathcal{H})$, we write $[A, B]:=A B-B A$. Let $\alpha:=\left\{\alpha_{i}\right\}_{i=0}^{\infty}$ be a weight sequence in the positive real number set $\mathbb{R}_{+}$. The weighted shift $W_{\alpha}$ acting on $\ell^{2}\left(\mathbb{N}_{0}\right)$, with an orthonormal basis $\left\{e_{i}\right\}_{i=0}^{\infty}$, is defined by $W_{\alpha} e_{i}=\alpha_{i} e_{i+1}$ for all $i \in \mathbb{N}_{0}$. Recall that a weighted shift $W_{\alpha}$ is cubically hyponormal if $W_{\alpha}+a W_{\alpha}^{2}+b W_{\alpha}^{3}$ is hyponormal for any $a, b \in \mathbb{C}([8])$, i.e.,

$$
D(a, b):=\left[\left(W_{\alpha}+a W_{\alpha}^{2}+b W_{\alpha}^{3}\right)^{*}, W_{\alpha}+a W_{\alpha}^{2}+b W_{\alpha}^{3}\right] \geq 0 \quad \text { for any } a, b \in \mathbb{C} .
$$

Let $P_{n}$ be the orthogonal projection onto the subspace $\vee_{i=0}^{n}\left\{e_{i}\right\}$. For $a, b \in \mathbb{C}$, we let

$$
\begin{aligned}
D_{n}(a, b) & =P_{n}\left[\left(W_{\alpha}+a W_{\alpha}^{2}+b W_{\alpha}^{3}\right)^{*}, W_{\alpha}+a W_{\alpha}^{2}+b W_{\alpha}^{3}\right] P_{n} \\
& =\left[\begin{array}{ccccccccc}
q_{0} & r_{0} & z_{0} & 0 & & & & & \\
\overline{r_{0}} & q_{1} & r_{1} & z_{1} & 0 & & & & \\
\overline{z_{0}} & \overline{r_{1}} & q_{2} & r_{2} & z_{2} & 0 & & & \\
0 & \overline{z_{1}} & \overline{r_{2}} & q_{3} & r_{3} & z_{3} & \ddots & & \\
& \ddots & \ddots & \ddots & \ddots & \ddots & \ddots & \ddots & \\
& & \ddots & \ddots & \ddots & \ddots & \ddots & \ddots & 0 \\
& & & \ddots & \ddots & \ddots & \ddots & \ddots & z_{n-2} \\
& & & & \ddots & \ddots & \ddots & \ddots & r_{n-1}
\end{array}\right],
\end{aligned}
$$

where

$$
\begin{aligned}
q_{n}:= & \left(\alpha_{n}^{2}-\alpha_{n-1}^{2}\right)+\left(\alpha_{n}^{2} \alpha_{n+1}^{2}-\alpha_{n-2}^{2} \alpha_{n-1}^{2}\right)|a|^{2} \\
& +\left(\alpha_{n}^{2} \alpha_{n+1}^{2} \alpha_{n+2}^{2}-\alpha_{n-3}^{2} \alpha_{n-2}^{2} \alpha_{n-1}^{2}\right)|b|^{2}, \\
r_{n}:= & \alpha_{n}\left(\alpha_{n+1}^{2}-\alpha_{n-1}^{2}\right) \bar{a}+\alpha_{n}\left(\alpha_{n+1}^{2} \alpha_{n+2}^{2}-\alpha_{n-1}^{2} \alpha_{n-2}^{2}\right) a \bar{b}, \\
z_{n}:= & \alpha_{n} \alpha_{n+1}\left(\alpha_{n+2}^{2}-\alpha_{n-1}^{2}\right) \bar{b} .
\end{aligned}
$$

Then it is obvious that $W_{\alpha}$ is cubically hyponormal if and only if the pentadiagonal matrix $D_{n}(a, b) \geq 0$ for any $a, b \in \mathbb{C}$ and any $n \in \mathbb{N}$. 
Definition 2.1. (i) A weighted shift $W_{\alpha}$ is semi-cubically hyponormal with type $I$ if $W_{\alpha}+s W_{\alpha}^{3}$ is hyponormal for any $s \in \mathbb{C}$.

(ii) A weighted shift $W_{\alpha}$ is semi-cubically hyponormal with type II if $W_{\alpha}^{2}+$ $s W_{\alpha}^{3}$ is hyponormal for any $s \in \mathbb{C}$.

(iii) A weighted shift $W_{\alpha}$ is almost-cubically hyponormal if it is semi-cubically hyponormal with types I and II.

We first construct formulas on type I (see [7]). Let $n$ be a fixed positive integer. For $s \in \mathbb{C}$, we let

$$
\begin{aligned}
& D_{m}^{[n]}(s)=P_{m}\left[\left(W_{\alpha}+s W_{\alpha}^{n}\right)^{*}, W_{\alpha}+s W_{\alpha}^{n}\right] P_{m} \\
& =\left[\begin{array}{cccccccc}
q_{n, 0} & 0 & \cdots & 0 & z_{n, 0} & 0 & & \\
0 & q_{n, 1} & \ddots & \ddots & 0 & z_{n, 1} & \ddots & \\
\vdots & \ddots & \ddots & \ddots & \ddots & \ddots & \ddots & 0 \\
0 & \ddots & \ddots & q_{n, n-2} & 0 & \ddots & \ddots & z_{n, m-n+1} \\
\bar{z}_{n, 0} & 0 & \ddots & 0 & q_{n, n-1} & 0 & \ddots & 0 \\
0 & \bar{z}_{n, 1} & \ddots & \ddots & 0 & \ddots & \ddots & \vdots \\
& \ddots & \ddots & 0 & \ddots & \ddots & q_{n, m-1} & 0 \\
& & 0 & \bar{z}_{n, m-n+1} & 0 & \cdots & 0 & q_{n, m}
\end{array}\right],
\end{aligned}
$$

where

$$
\begin{aligned}
q_{n, k} & =u_{n, k}+v_{n, k}|s|^{2} \\
z_{n, k} & =\sqrt{w_{n, k}} \bar{s} \\
u_{n, k} & =\alpha_{k}^{2}-\alpha_{k-1}^{2} \\
v_{n, k} & =\alpha_{k}^{2} \alpha_{k+1}^{2} \cdots \alpha_{k+n-1}^{2}-\alpha_{k-n}^{2} \alpha_{k-2}^{2} \cdots \alpha_{k-1}^{2}, \\
w_{n, k} & =\alpha_{k}^{2} \alpha_{k+1}^{2} \cdots \alpha_{k+n-2}^{2}\left(\alpha_{k+n-1}^{2}-\alpha_{k-1}^{2}\right)^{2}
\end{aligned}
$$

with $\alpha_{-1}=\alpha_{-2}=\cdots=\alpha_{-n}=0$ (for our convenience). Then it is obvious that $W_{\alpha}$ is semi-weakly $n$-hyponormal if and only if $D_{m}^{[n]}(s) \geq 0$ for all $s \in \mathbb{C}, m \in \mathbb{N}$. To detect $D_{m}^{[n]}(s) \geq 0$ for all $s \in \mathbb{C}$, we usually use the Nested Determinant Test $([3])$. Now we consider $d_{j}^{[n]}:=d_{j}^{[n]}(s)=\operatorname{det} D_{j}^{[n]}(s)$ for $1 \leq j \leq m$.

By changing the standard basis of $\mathbb{C}^{n+1}$, we have the following lemma.

Lemma 2.2 ([7, Lemma 2.1]). Let $n \geq 2$ be a fixed positive integer. For $m \in \mathbb{N}$, if two non-negative integers $k$ and $j$ satisfy $m=(n-1) k+j$, i.e., $m \equiv j(\bmod n-1)$, then $D_{m}^{[n]}(s)$ is unitarily equivalent to $\left[\oplus_{i=0}^{j} D_{k, 1}^{[n]}(i, j)\right] \oplus$ 
$\left[\oplus_{i=j+1}^{n-2} D_{k, 2}^{[n]}(i, j)\right]$, where

(2.1)

$$
D_{k, 1}^{[n]}(i, j)=\left[\begin{array}{ccccc}
q_{n, j-i} & z_{n, j-i} & 0 & & \\
\bar{z}_{n, j-i} & q_{n,(n-1)+j-i} & \ddots & \ddots & 0 \\
0 & \bar{z}_{n,(n-1)+j-i} & \ddots & \ddots & \\
& \ddots & \ddots & q_{n,(k-1)(n-1)+j-i} & z_{n,(k-1)(n-1)+j-i}
\end{array}\right]
$$

for $0 \leq i \leq j$, and

$$
D_{k, 2}^{[n]}(i, j)=\left[\begin{array}{ccccc}
q_{n,(n-1)+j-i} & z_{n,(n-1)+j-i} & 0 & & \\
\bar{z}_{n,(n-1)+j-i} & q_{n, 2(n-1)+j-i} & \ddots & & \\
0 & \bar{z}_{n, 2(n-1)+j-i} & \ddots & \ddots & 0 \\
& \ddots & \ddots & q_{n,(k-1)(n-1)+j-i} & z_{n,(k-1)(n-1)+j-i} \\
& & 0 & \bar{z}_{n,(k-1)(n-1)+j-i} & q_{n, k(n-1)+j-i}
\end{array}\right]
$$

for $j+1 \leq i \leq n-2$. Therefore, we have that

$$
\operatorname{det} D_{m}^{[n]}(s)=\prod_{i=0}^{j} \operatorname{det} D_{k, 1}^{[n]}(i, j) \cdot \prod_{i=j+1}^{n-2} \operatorname{det} D_{k, 2}^{[n]}(i, j) .
$$

Moreover, a weighted shift $W_{\alpha}$ is semi-weakly $n$-hyponormal if and only if $D_{k, 1}^{[n]}(i, j) \geq 0(0 \leq i \leq j)$ and $D_{k, 2}^{[n]}(i, j) \geq 0(j+1 \leq i \leq n-2)$.

As a special example of Lemma 2.2, we consider here the case of $n=3$. This case is $j=0$ or 1 obviously. Lemma 2.2 implies that the matrix $D_{m}^{[3]}(s)$ induces the following two cases:

$$
\operatorname{det} D_{m}^{[3]}(s)=\left\{\begin{array}{cl}
\operatorname{det} D_{2 k}^{[3]}(s)=\operatorname{det} D_{k, 1}^{[n]}(0,0) \cdot \operatorname{det} D_{k, 2}^{[n]}(1,0) & \text { if } j=0 \\
\operatorname{det} D_{2 k+1}^{[3]}(s)=\operatorname{det} D_{k, 1}^{[n]}(0,1) \cdot \operatorname{det} D_{k, 1}^{[n]}(1,1) & \text { if } j=1 .
\end{array}\right.
$$

Since

$$
D_{k, 1}^{[n]}(0,0)=D_{k, 1}^{[n]}(1,1)=\left[\begin{array}{llll}
q_{3,0} & z_{3,0} & 0 & \\
\bar{z}_{3,0} & q_{3,2} & z_{3,2} & \\
0 & \bar{z}_{3,2} & \ddots & \ddots \\
& & \ddots & q_{3,2 k}
\end{array}\right]
$$

and $D_{k, 2}^{[n]}(1,0)$ is a submatrix of $D_{k, 1}^{[n]}(0,1)$, if the following two matrices

$$
D_{k, 1}^{[3]}(0,0)=\left[\begin{array}{llll}
q_{3,0} & z_{3,0} & 0 & \\
\bar{z}_{3,0} & q_{3,2} & z_{3,2} & \\
0 & \bar{z}_{3,2} & \ddots & \ddots \\
& & \ddots & q_{3,2 k}
\end{array}\right], D_{k, 1}^{[3]}(0,1)=\left[\begin{array}{llll}
q_{3,1} & z_{3,1} & 0 & \\
\bar{z}_{3,1} & q_{3,3} & z_{3,3} & \\
0 & \bar{z}_{3,3} & \ddots & \\
& & & q_{3,2 k+1}
\end{array}\right]
$$

are all positive, then $W_{\alpha}$ is semi-cubically hyponormal with type I. 
We construct formulas on type II. For $s \in \mathbb{C}$, we have

$$
\begin{aligned}
M_{n}(s): & P_{n}\left[\left(W_{\alpha}^{2}+s W_{\alpha}^{3}\right)^{*},\left(W_{\alpha}^{2}+s W_{\alpha}^{3}\right)\right] P_{n} \\
& =\left[\begin{array}{ccccccc}
\omega_{0} & \bar{\phi}_{0} & 0 & & & & \\
\phi_{0} & \omega_{1} & \bar{\phi}_{1} & 0 & & & \\
0 & \phi_{1} & \omega_{2} & \bar{\phi}_{2} & 0 & & \\
& 0 & \phi_{2} & \omega_{3} & \ddots & \ddots & \\
& & \ddots & \ddots & \ddots & \ddots & 0 \\
& & & \ddots & \ddots & \ddots & \bar{\phi}_{n-1} \\
& & & & 0 & \phi_{n-1} & \omega_{n}
\end{array}\right],
\end{aligned}
$$

where

$\omega_{k}:=\xi_{k}+|s|^{2} \eta_{k}, \quad \phi_{k}:=s \sqrt{\delta_{k}}, \quad \xi_{k}:=\alpha_{k}^{2} \alpha_{k+1}^{2}-\alpha_{k-2}^{2} \alpha_{k-1}^{2}$,

$\eta_{k}:=\alpha_{k}^{2} \alpha_{k+1}^{2} \alpha_{k+2}^{2}-\alpha_{k-3}^{2} \alpha_{k-2}^{2} \alpha_{k-1}^{2}, \delta_{k}:=\alpha_{k}^{2}\left(\alpha_{k+1}^{2} \alpha_{k+2}^{2}-\alpha_{k-2}^{2} \alpha_{k-1}^{2}\right)^{2}(k \geq 0)$

and $\alpha_{-1}=\alpha_{-2}=\alpha_{-3}:=0$. Put $\sigma_{n}:=\sigma_{n}(\cdot)=\operatorname{det}\left(M_{n}(\cdot)\right)$. Then

$$
\sigma_{0}=\omega_{0}, \sigma_{1}=\omega_{0} \omega_{1}-\left|\phi_{0}\right|^{2}, \sigma_{n+2}=\omega_{n+2} \sigma_{n+1}-\left|\phi_{n+1}\right|^{2} \sigma_{n} \quad(n \geq 0)
$$

and $\sigma_{n}$ is actually a polynomial in $t:=|s|^{2}$ of degree $n+1$, with Maclaurin's expansion $\sigma_{n}(t):=\sum_{i=0}^{n+1} c(n, i) t^{i}$. It follows from the similar method in [2] that

(i) $c(0,0)=\xi_{0}, \quad c(0,1)=\eta_{0}$

(ii) $c(1,0)=\xi_{1} \xi_{0}, \quad c(1,1)=\xi_{1} \eta_{0}+\xi_{0} \eta_{1}, \quad c(1,2)=\eta_{1} \eta_{0}$

(iii) $c(n+2, i)=\xi_{n+2} c(n+1, i)+\eta_{n+2} c(n+1, i-1)-\delta_{n+1} c(n, i-1)$ $(n \geq 0, \quad 0 \leq i \leq n+3)$;

(iv) $c(n, n+1)=\eta_{0} \eta_{1} \cdots \eta_{n}>0$ and $c(n, 0)=\xi_{0} \xi_{1} \cdots \xi_{n}>0$ for all $n \in \mathbb{N}_{0}$.

\section{Main results}

It is well known that if a weighted shift $W_{\alpha}$ is quadratically hyponormal with first two equal weights, it is not necessarily flat ([2]). For example, if $\alpha: \sqrt{\frac{2}{3}}, \sqrt{\frac{2}{3}}, \sqrt{\frac{3}{4}}, \sqrt{\frac{4}{5}}, \sqrt{\frac{5}{6}}, \ldots$, then the associated weighted shift $W_{\alpha}$ is quadratically hyponormal. And the author in [1] showed the following:

Proposition 3.1 ([1, Theorem 1]). Let $W_{\alpha}$ be a quadratically hyponormal weighted shift with $\alpha=\left\{\alpha_{n}\right\}_{n=0}^{\infty}$. If $\alpha_{n}=\alpha_{n+1}$ for some $n \geq 1$, then $W_{\alpha}$ is flat, i.e., $\alpha_{1}=\alpha_{2}=\cdots$.

First, we give the following lemma.

Lemma 3.2. Let $f(x)=a_{0} x^{3}+a_{1} x^{2}+a_{2} x+a_{3}$ be any cubic real polynomial. Then $f(x) \geq 0$ for all $x \geq 0$ if and only if it holds one of the following cases:

(1) $a_{0}>0, a_{1}>0, a_{2} \geq 0, a_{3} \geq 0$

(2) $a_{0}>0, a_{3} \geq 0$ and $a_{1}^{2}-3 a_{0} a_{2} \leq 0$; 
(3) $a_{0}>0, a_{3} \geq 0, a_{2}<0,27 a_{0}^{2} a_{3}+2 a_{1}^{3}-9 a_{0} a_{1} a_{2} \geq 0$ and

$$
4 a_{0} a_{2}^{3}+4 a_{1}^{3} a_{3}+27 a_{0}^{2} a_{3}^{2}-a_{1}^{2} a_{2}^{2}-18 a_{0} a_{1} a_{2} a_{3} \geq 0 ;
$$

(4) $a_{0}>0, a_{3} \geq 0, a_{1} \leq 0, a_{2} \geq 0, a_{1}^{2}-3 a_{0} a_{2}>0,27 a_{0}^{2} a_{3}+2 a_{1}^{3}-9 a_{0} a_{1} a_{2} \geq$ 0 and

$$
4 a_{0} a_{2}^{3}+4 a_{1}^{3} a_{3}+27 a_{0}^{2} a_{3}^{2}-a_{1}^{2} a_{2}^{2}-18 a_{0} a_{1} a_{2} a_{3} \geq 0 .
$$

Proof. It is easy to see that one of the necessary conditions is $a_{0}>0, a_{3} \geq 0$. From a calculation, we have $f^{\prime}(x)=3 a_{0} x^{2}+2 a_{1} x+a_{2}$. If $a_{1}^{2}-3 a_{0} a_{2} \leq 0$, using $a_{0}>0$, then $f^{\prime}(x) \geq 0$ for all $x \geq 0$, which implies that $f(x) \geq 0$ for all $x \geq 0$. This is the case of $(2)$.

If $a_{0}>0, a_{3} \geq 0$ and $a_{1}>0, a_{2} \geq 0$, then it is the case of (1).

Now we suppose that $a_{1}^{2}-3 a_{0} a_{2}>0, a_{0}>0$ and $a_{3} \geq 0$. Denote $x_{2}:=$ $\frac{-a_{1}+\sqrt{a_{1}^{2}-3 a_{0} a_{2}}}{3 a_{0}}$ be the largest root of the equation $f^{\prime}(x)=0$. We consider two cases of $x_{2}<0$ and $x_{2} \geq 0$. For the case of $x_{2}<0$, it follows from $a_{0}>0$ that $f^{\prime}(x) \geq 0$ for all $x \geq 0$. Using the condition $a_{3} \geq 0$, we have $f(x) \geq 0(x \geq 0)$. If $x_{2} \geq 0$, i.e., $a_{2}<0$ or $a_{1} \leq 0, a_{2} \geq 0$, which is the case (3) or (4) resp., then from Fermat's theorem, $f\left(x_{2}\right)$ is the local minimum of $f(x)$ for $x \geq 0$. From simple computation, we have

$$
f\left(x_{2}\right)=\frac{1}{27} \frac{27 a_{0}^{2} a_{3}+2 a_{1}^{3}-9 a_{0} a_{1} a_{2}-2\left(a_{1}^{2}-3 a_{0} a_{2}\right)^{3 / 2}}{a_{0}^{2}} .
$$

Hence $f\left(x_{2}\right) \geq 0$ is equivalent to $27 a_{0}^{2} a_{3}+2 a_{1}^{3}-9 a_{0} a_{1} a_{2} \geq 0$, and

$$
2\left(a_{1}^{2}-3 a_{0} a_{2}\right)^{3 / 2} \leq 27 a_{0}^{2} a_{3}+2 a_{1}^{3}-9 a_{0} a_{1} a_{2},
$$

that is,

$$
4 a_{0} a_{2}^{3}+4 a_{1}^{3} a_{3}+27 a_{0}^{2} a_{3}^{2}-a_{1}^{2} a_{2}^{2}-18 a_{0} a_{1} a_{2} a_{3} \geq 0 .
$$

Thus we have the lemma.

The following result shows that the cubically hyponormal weighted shift with first two equal weights has flatness. Hence, the nonsubnormal but cubically hyponormal weighted shift operator should have strictly increasing weights.

Theorem 3.3. If a weighted shift $W_{\alpha}$ is cubically hyponormal with $\alpha_{0}=\alpha_{1}$, then $W_{\alpha}$ is flat.

Proof. Assume that $\left\{\alpha_{n}\right\}_{n=0}^{\infty}$ is nondecreasing, and without loss of generality, let $\alpha_{0}=\alpha_{1}=1$. We claim that $\alpha_{2}=1$.

Suppose that $\alpha_{2}>1$. Since $W_{\alpha}$ is cubically hyponormal, we must have

$$
\begin{aligned}
& f(a, b):=\operatorname{det} D_{2}(a, b) \\
& =\left|\begin{array}{ccc}
1+a^{2}+\alpha_{2}^{2} b^{2} & a+\alpha_{2}^{2} a b & \alpha_{2}^{2} b \\
a+\alpha_{2}^{2} a b & \alpha_{2}^{2} a^{2}+\alpha_{2}^{2} \alpha_{3}^{2} b^{2} & a\left(\alpha_{2}^{2}-1\right)+a b \alpha_{2}^{2} \alpha_{3}^{2} \\
\alpha_{2}^{2} b & a\left(\alpha_{2}^{2}-1\right)+a b \alpha_{2}^{2} \alpha_{3}^{2} & \alpha_{2}^{2}-1+a^{2}\left(\alpha_{2}^{2} \alpha_{3}^{2}-1\right)+b^{2} \alpha_{2}^{2} \alpha_{3}^{2} \alpha_{4}^{2}
\end{array}\right| \\
& =a_{0} a^{6}+a_{1} a^{4}+a_{2} a^{2}+a_{3} \geq 0,
\end{aligned}
$$


for any $a, b \in \mathbb{R}$, where

$$
\begin{aligned}
a_{0}= & \alpha_{2}^{2}\left(\alpha_{2}^{2} \alpha_{3}^{2}-1\right), \\
a_{1}= & \alpha_{2}^{2} \alpha_{3}^{2}\left(\alpha_{2}^{2} \alpha_{4}^{2}-1\right) b^{2}-2 \alpha_{2}^{2}\left(-\alpha_{3}^{2}+2 \alpha_{2}^{2} \alpha_{3}^{2}-1\right) b+\alpha_{2}^{2} \alpha_{3}^{2}\left(\alpha_{2}^{2}-1\right), \\
a_{2}= & \alpha_{2}^{4} \alpha_{3}^{2}\left(\alpha_{3}^{2} \alpha_{4}^{2}-1\right) b^{4}-2 \alpha_{2}^{4} \alpha_{3}^{2}\left(\alpha_{4}^{2}-1\right) b^{3} \\
& +\alpha_{2}^{2}\left(-2 \alpha_{3}^{2}+3 \alpha_{2}^{2} \alpha_{3}^{2}-\alpha_{3}^{2} \alpha_{4}^{2}+\alpha_{2}^{2} \alpha_{3}^{2} \alpha_{4}^{2}-1\right) b^{2}-2 \alpha_{2}^{2} \alpha_{3}^{2}\left(\alpha_{2}^{2}-1\right) b, \\
a_{3}= & \left(\alpha_{2}^{6} \alpha_{3}^{4} \alpha_{4}^{2}\right) b^{6}+\alpha_{2}^{4} \alpha_{3}^{2}\left(\alpha_{3}^{2} \alpha_{4}^{2}-1\right) b^{4}+\alpha_{2}^{2} \alpha_{3}^{2}\left(\alpha_{2}^{2}-1\right) b^{2} .
\end{aligned}
$$

Since $a_{0}>0, a_{3} \geq 0$, by Lemma 3.2, if $f(a, b)=a_{0} a^{6}+a_{1} a^{4}+a_{2} a^{2}+a_{3} \geq 0$, for any $a, b \in \mathbb{R}$, then either one of the followings holds:

(i) $a_{2} \geq 0$. But $a_{2}<0$, if $b$ is positive infinitesimal, so it is a contradiction.

(ii) Since

$$
\Delta_{1}:=a_{1}^{2}-3 a_{0} a_{2}=(\cdot) b^{4}+(\cdot) b^{3}+(\cdot) b^{2}+(\cdot) b+\alpha_{2}^{4} \alpha_{3}^{4}\left(\alpha_{2}^{2}-1\right)^{2},
$$

thus $\Delta_{1} \leq 0$ implies that $\alpha_{2}^{4} \alpha_{3}^{4}\left(\alpha_{2}^{2}-1\right)^{2} \leq 0$ by taking $b=0$, which induces a contradiction.

(iii) Since

$$
\begin{aligned}
\Delta_{2} & :=4 a_{0} a_{2}^{3}+4 a_{1}^{3} a_{3}+27 a_{0}^{2} a_{3}^{2}-a_{1}^{2} a_{2}^{2}-18 a_{0} a_{1} a_{2} a_{3} \\
& =(\cdot) b^{12}+(\cdot) b^{11}+(\cdot) b^{10}+\cdots+(\cdot) b^{4}+4 \alpha_{2}^{8} \alpha_{3}^{8} \alpha_{4}^{2}\left(\alpha_{2}-1\right)^{4}\left(\alpha_{2}+1\right)^{4} b^{3},
\end{aligned}
$$

if $b$ is negative infinitesimal, then $\Delta_{2}<0$. It is also a contradiction. Hence we must have $\alpha_{2}=1$. Since cubic hyponormality implies quadratic hyponormality, by Proposition 3.1, we know that $W_{\alpha}$ is flat.

By Proposition 3.1 and Theorem 3.3, we have the following results.

Corollary 3.4. Let $W_{\alpha}$ be a cubically hyponormal weighted shift with $\alpha=$ $\left\{\alpha_{n}\right\}_{n=0}^{\infty}$. If $\alpha_{n}=\alpha_{n+1}$ for some $n \geq 0$, then $W_{\alpha}$ is flat.

Corollary 3.5. Let $W_{\alpha}$ be a weighted shift with a weight sequence $\alpha$, where

$$
\alpha: \alpha_{0}=\alpha_{1}=\sqrt{\frac{2}{3}}, \quad \text { and } \quad \alpha_{k}=\sqrt{\frac{k+1}{k+2}}, \quad k=2,3, \ldots
$$

Then $W_{\alpha}$ is not cubically hyponormal.

Proposition 3.6. Let $W_{\alpha(x, x)}$ be a weighted shift with $\alpha(x, x)$, where

$$
\alpha(x, x): \alpha_{0}=\alpha_{1}=\sqrt{x}, \quad \text { and } \alpha_{k}=\sqrt{\frac{k+1}{k+2}}, \quad k=2,3, \ldots
$$

If $\frac{1}{19}(14-\sqrt{6}) \leq x \leq \sqrt{5}-\frac{3}{2}$, then $W_{\alpha(x, x)}$ is semi-cubically hyponormal with type I. In particular, $W_{\alpha\left(\frac{2}{3}, \frac{2}{3}\right)}$ is semi-cubically hyponormal with type $I$.

Proof. See [7, Corollary 3.7].

Proposition 3.7. Let $W_{\alpha(x)}$ be a weighted shift with $\alpha(x)$, where

$$
\alpha(x): \alpha_{0}=\sqrt{x} \text { and } \alpha_{k}=\sqrt{\frac{k+1}{k+2}}, \quad k=1,2, \ldots
$$

If $0<x<\frac{9}{10}$, then $W_{\alpha(x)}$ is semi-cubically hyponormal with type II. 
Proposition 3.7 can be proved by some computations as following steps.

Lemma 3.8. $\xi_{n+1} \eta_{n}=\delta_{n}$ for $n \geq 4$.

Proof. From simple computations, we can have

$$
\begin{aligned}
& \xi_{n}=\alpha_{n}^{2} \alpha_{n+1}^{2}-\alpha_{n-2}^{2} \alpha_{n-1}^{2}=\frac{4}{(n+1)(n+3)} \quad(n \geq 3) \\
& \eta_{n}=\alpha_{n}^{2} \alpha_{n+1}^{2} \alpha_{n+2}^{2}-\alpha_{n-3}^{2} \alpha_{n-2}^{2} \alpha_{n-1}^{2}=\frac{9}{(n+1)(n+4)} \quad(n \geq 4) \\
& \delta_{n}=\alpha_{n}^{2}\left(\alpha_{n+1}^{2} \alpha_{n+2}^{2}-\alpha_{n-2}^{2} \alpha_{n-1}^{2}\right)^{2}=\frac{36}{(n+1)(n+2)(n+4)^{2}} \quad(n \geq 3)
\end{aligned}
$$

which induces this lemma.

Lemma 3.9. For $n \geq 5$ and $i \geq 1$, we have $c(n, i)=\eta_{n} c(n-1, i-1)+\left(\xi_{n} \cdots \xi_{5}\right) h_{i}$ with $h_{i}:=\xi_{4} c(3, i)-\delta_{3} c(2, i-1)$.

Proof. Using Lemma 3.8, we have the result by similar to the proof of the claim in $[2$, page 64$]$.

Now we consider the sequence $\alpha(x)$ as in (3.3), we can obtain

$$
\begin{aligned}
& \sigma_{0}(t)=\frac{x}{6}(3 t+4), \quad \sigma_{1}(t)=\frac{x}{15}\left(4 t+3 t^{2}+5\right), \\
& \sigma_{2}(t)=\frac{1}{90} x\left(9 t^{3}+(12-12 x) t^{2}+(15-16 x) t+(18-20 x)\right), \\
& \sigma_{3}(t)=-\frac{x}{3780}\left[(189 x-216) t^{4}+(36 x-99) t^{3}+(111 x-108) t^{2}\right. \\
&\quad+(130 x-117) t+140 x-126], \\
& \sigma_{4}(t)=-\frac{x}{151200}\left[(1701 x-1944) t^{5}+(1188 x-1323) t^{4}+(423 x-684) t^{3}\right. \\
&\left.\quad+(690 x-621) t^{2}+(620 x-558) t+640 x-576\right], \\
& \sigma_{5}(t)=-\frac{x}{907200}\left[(1701 x-1944) t^{6}+(1188 x-1323) t^{5}+(855 x-900) t^{4}\right. \\
&\left.+(402 x-477) t^{3}+(380 x-342) t^{2}+(320 x-288) t+320 x-288\right],
\end{aligned}
$$

and

$$
\begin{aligned}
& h_{1}=\frac{2 x}{4725}(10 x-9), h_{2}=\frac{x}{3150}(10 x-9), h_{3}=\frac{x}{525}(2 x-1), \\
& h_{4}=-\frac{x}{350}(2 x-1), h_{i}=0 \quad \text { for all } i \geq 5 .
\end{aligned}
$$


Proof of Proposition 3.7. For $0<x<\frac{9}{10}$, it is easy to see that each of the coefficients of $\sigma_{i}(t)(i=0,1,2,3,4,5)$ is positive, furthermore we have

$$
\begin{aligned}
& c(6,2)=-\frac{17}{5953500} x(10 x-9)>0, \\
& c(6,3)=-\frac{1}{31752000} x(1070 x-1219)>0, \\
& c(6,4)=-\frac{1}{21168000} x(1846 x-1751)>0, \\
& c(7,3)=-\frac{1}{174636000} x(334 x-371)>0, \\
& c(7,4)=-\frac{1}{931392000} x(4618 x-4361)>0, \\
& c(8,4)=-\frac{1}{698544000} x(154 x-145)>0 .
\end{aligned}
$$

Let

$$
\begin{aligned}
\rho_{1} & =-\frac{2}{4725} \frac{x(5 n+11)}{(n+1)(n+3)(n+4)}, \\
\rho_{2} & =-\frac{2}{1575} \frac{x(11 n+2)}{n(n+1)(n+2)(n+3)(n+4)}, \\
\rho_{3} & =-\frac{2}{525} \frac{x(266 n-526)}{n(n-1)(n+1)^{2}(n+2)(n+3)(n+4)}, \\
\rho_{4} & =-\frac{2}{175} \frac{x(926 n-1864)}{n^{2}(n-1)(n+1)^{2}(n-2)(n+2)(n+3)(n+4)},
\end{aligned}
$$

we can see that $\rho_{i}<0(i=1,2,3,4)$ for $n \geq 3$. Since $0<x<\frac{9}{10}, \frac{265 n-371}{266 n-526}>\frac{9}{10}$ and $\frac{859 n-1652}{926 n-1864}>\frac{9}{10}$, using Lemma 3.9, we have

$$
\begin{aligned}
& c(n, 1)=\rho_{1}(10 x-9)\left(\xi_{n-1} \cdots \xi_{5}\right)>0 \text { for } n \geq 6, \\
& c(n, 2)=\rho_{2}(10 x-9)\left(\xi_{n-2} \cdots \xi_{5}\right)>0 \text { for } n \geq 7, \\
& c(n, 3)=\rho_{3}\left(x-\frac{265 n-371}{266 n-526}\right)\left(\xi_{n-3} \cdots \xi_{5}\right)>0 \text { for } n \geq 8, \\
& c(n, 4)=\rho_{4}\left(x-\frac{859 n-1652}{926 n-1864}\right)\left(\xi_{n-4} \cdots \xi_{5}\right)>0 \text { for } n \geq 9 .
\end{aligned}
$$

This concludes the proof.

Finally, by Corollary 3.5, Proposition 3.6 and Proposition 3.7, we obtain:

Theorem 3.10. Let $W_{\alpha}$ be a weighted shift with a sequence $\alpha$ as in (3.1). Then $W_{\alpha}$ is almost-cubically hyponormal but not cubically hyponormal.

\section{References}

[1] Y. Choi, A propagation of quadratically hyponormal weighted shifts, Bull. Korean Math. Soc. 37 (2000), no. 2, 347-352. 
[2] R. Curto, Quadratically hyponormal weighted shifts, Integ. Equ. Oper. Theory 13 (1990), no. $1,49-66$.

[3] R. Curto and L. Fialkow, Recursively generated weighted shifts and the subnormal completion problem, Integ. Equ. Oper. Theory 17 (1993), no. 2, 202-246.

[4] _ Recursively generated weighted shifts and the subnormal completion problem. II, Integ. Equ. Oper. Theory 18 (1994), no. 4, 369-426.

[5] R. Curto and I. Jung, Quadratically hyponormal weighted shifts with two equal weights, Integ. Equ. Oper. Theory 37 (2000), no. 2, 208-231.

[6] R. Curto and M. Putinar, Nearly subnormal operators and moment problems, J. Funct. Anal. 115 (1993), no. 2, 480-497.

[7] Y. Do, G. Exner, I. Jung, and C. Li, On semi-weakly n-hyponormal weighted shifts, Integ. Equ. Oper. Theory 73 (2012), no. 1, 93-106.

[8] I. Jung and S. Park, Cubically hyponormal weighted shifts and their examples, J. Math. Anal. Appl. 247 (2000), no. 2, 557-569.

[9] J. Stampfli, Which weighted shifts are subnormal, Pacific J. Math. 17 (1966), 367-379.

[10] Wolfram Research, Inc. Mathematica, Version 8.0, Wolfram Research, Champaign, 2010.

Chunji Li

Institute of System Science

NORTHEASTERN UNIVERSITY

Shenyang 110004, P. R. China

E-mail address: chunjili2000@aliyun.com

Muneo Chō

Department of Mathematics

FACUlTy OF SCIENCE

KANAGAWA UNIVERSITY

HiRATSUKA 259-1293, JAPAN

E-mail address: chiyom01@kanagawa-u.ac.jp

Mi Ryeong Lee

Institute of Liberal Education

CAtholic University of DAegu

Gyeongbuk 712-702, Korea

E-mail address: leemr@cu.ac.kr 